\title{
[18] ANÁLISIS DE LA INTRODUCCIÓN DE LA SOSTENIBILIDAD EN LA ENSEÑANZA SUPERIOR EN EUROPA: COMPROMISOS INSTITUCIONALES Y PROPUESTAS CURRICULARES ${ }^{1}$
}

\author{
UII, M.A $A^{1}$; Martínez Agut, M. $P^{2}$.; Piñero, $A^{3}$; Aznar Minguet, $P^{2}$. \\ Universidad de València \\ ${ }^{1}$ Dpto. Bioquímica y Biología Molecular. angels.ull@uv.es \\ 2 Dpto.Teoría de la Educación.M.pilar.martinez@uv.es, pilar.aznar@uv.es \\ ${ }^{3}$ Dpto. Sociología y Antropología Social. Albert.pinero@uv.es
}

[Recibido en Diciembre de 2009, aceptado en Enero de 2010]

\begin{abstract}
RESUMEN
En el actual contexto del proceso de armonización de los planes de estudio universitarios y la convergencia de las universidades europeas, la Declaración de Bolonia ya recoge la idea de que hay que hacer de la educación superior un área sostenible. Desde esta óptica, en este artículo, se analizan, por una parte, las disposiciones legales que se vienen estableciendo para comprobar si las referencias a la inclusión de criterios de sostenibilidad se traducen en la elaboración de los nuevos planes de estudios en las universidades europeas; y por otra parte se analizan las actividades de las redes de colaboración (Copernicus, HBO, ULSF, ACES,...) existentes entre las universidades europeas, para la introducción de la sostenibilidad en los currícula de la educación superior. Consideramos que este análisis puede contribuir a una valoración de las acciones realizadas a nivel de estudios superiores, en el contexto de la Década de la Educación para el Desarrollo Sostenible impulsada por la UNESCO.
\end{abstract}

Palabras clave: Sostenibilidad; Educación Superior; ámbito europeo.

\section{INTRODUCCIÓN: UNIVERSIDAD Y SOSTENIBILIDAD}

La UNESCO, en 1998, apuntaba que cada vez más, las universidades están llamadas a desempeñar una función de liderazgo en el desarrollo de formas de educación interdisciplinarias y transdisciplinarias y éticamente orientadas, a fin de idear soluciones para los problemas vinculados al desarrollo sostenible.

Las universidades no se constituyen tan solo en centros de generación de conocimiento, sino que a través de la formación de los estudiantes, la investigación, la promoción de una conciencia crítica, etc..., asumen un rol de responsabilidad sin precedentes en la historia de los estudios superiores, de difusión de conocimientos,

\footnotetext{
${ }^{1}$ Este trabajo se ha realizado dentro del Proyecto de Investigación SEJ2007-67063/EDUC subvencionado por el Ministerio de Ciencia y Tecnología
} 
valores, actitudes y comportamientos favorables hacia un desarrollo humano ambientalmente sostenible a aplicar por los nuevos titulados universitarios en el ejercicio de sus respectivas profesiones.

Aunque realmente en el ámbito universitario la educación para la sostenibilidad se ha iniciado en la década de los noventa, existen varias referencias a nivel nacional e internacional de la coordinación de las universidades interesadas en la introducción de la sostenibilidad en la docencia, la investigación la gestión y las relaciones con la sociedad que las rodea, que se han concretado en diversas declaraciones internacionales como puede verse en la tabla siguiente y cuyas páginas web de referencia pueden consultarse en la bibliografía (Cuadro 1 ).

\begin{tabular}{|c|c|c|}
\hline Año & Nonbre de la Declaración & Institución promotora y objetivos \\
\hline 1990 & La Declaración de Talloires & $\begin{array}{l}\text { Firmada en Paris. En la creación de la Asociación de } \\
\text { University Leaders for a Sustainable Future (ULSF). }\end{array}$ \\
\hline 1991 & $\begin{array}{l}\text { La Declaración de Halifax } \\
\text { "Creando un futuro común: } \\
\text { un plan de acción para las } \\
\text { universidades". }\end{array}$ & $\begin{array}{l}\text { Firmada durante la Conferencia de Acción de las } \\
\text { universidades hacia un desarrollo sostenible } \\
\text { organizada por la International Association of } \\
\text { Universities (IAU). }\end{array}$ \\
\hline 1993 & La declaración de Swansea & $\begin{array}{l}\text { Refleja el compromiso de las Universidades de la } \\
\text { Commonwealth para dar respuestas al reto de la } \\
\text { sostenibilidad }\end{array}$ \\
\hline 1993 & $\begin{array}{llr}\text { La Carta } & \text { de } & \text { las } \\
\text { Universidades por } & \text { el } \\
\text { Desarrollo sostenible } & \end{array}$ & $\begin{array}{l}\text { Conferencia de Rectores Europeos }(\mathrm{CRE})^{2} \quad \text { (El } \\
\text { Proyecto Copernicus ) }\end{array}$ \\
\hline 1997 & La Declaración de Lüneburg & $\begin{array}{l}\text { Educación Superior para el desarrollo sostenible: } \\
\text { promoviendo el desarrollo de una Agenda } 21 \text { para la } \\
\text { universidad }\end{array}$ \\
\hline 2003 & 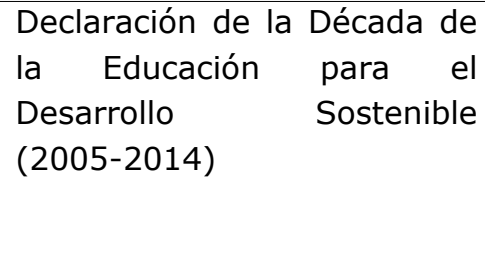 & $\begin{array}{l}\text { UNESCO. (El propósito es construir un mundo en el } \\
\text { que cada una tenga acceso igualitario a la educación } \\
\text { en valores, comportamientos y estilos de vida } \\
\text { coherentes con un futuro sostenible hacia una } \\
\text { transformación positiva de la sociedad (Ver UNESCO } \\
1998,2005 \text { ). }\end{array}$ \\
\hline 2005 & $\begin{array}{l}\text { Lübeck Declaration: } \\
\text { University and Sustainability }\end{array}$ & Universidades de habla alemana \\
\hline
\end{tabular}

\section{Cuadro 1}

${ }^{2}$ El Proyecto COPERNICUS; se dirige a implementar la perspectiva de la sostenibilidad en todo el sistema universitario, estimular y coordinar proyectos de investigación intedisciplinarios, estrechar las relaciones de la universidad con otros sectores sociales y promocionar la formación de todos los titulados universitarios en competencias, no sólo científicas y técnicas, sino también sostenibilizadoras para que en el posterior desempeño de sus funciones profesionales tomen decisiones y realicen sus acciones de acuerdo a criterios de sostenibilidad. 


\begin{tabular}{|c|l|l|l|}
\hline Año & Nonbre de la Declaración & \multicolumn{1}{|c|}{ Institución promotora y objetivos } \\
\hline 2008 & $\begin{array}{l}\text { La educación superior en } \\
\text { el mundo: nuevos retos y } \\
\text { roles emergentes para el } \\
\text { desarrollo humano y } \\
\text { social. }\end{array}$ & $\begin{array}{l}\text { Global University Network for Innovation } \\
\text { (GUNI). Barcelona Meeting (UPC). }\end{array}$ & \\
\hline 2009 & Declaración de Bonn & $\begin{array}{l}\text { Conferencia Mundial de UNESCO sobre } \\
\text { Educación para el Desarrollo Sostenible }\end{array}$ & \\
\hline
\end{tabular}

\section{Cuadro 1 (Continuación)}

\section{MARCO TEÓRICO}

\section{Educación para la sostenibilidad en los estudios superiores}

Un factor decisivo para conseguir una cultura de la sostenibilidad es la formación universitaria de los futuros profesionales (Clugston, 2002).Todos los alumnos universitarios tendrían que formarse en sus campos de especialización de acuerdo con criterios y valores ambientales y sostenibles. Los universitarios habrían de adquirir una comprensión central de la visión de la sostenibilidad, de tal forma que en el futuro pudiesen considerar esta perspectiva en sus actividades profesionales. Para ello debemos fijarnos unos objetivos a alcanzar, entre los cuáles cabría subrayar:

- Reforzar las competencias ambientales de los/las profesionales formados en la universidad (Tobón, 2004).

- Promover incentivos en la docencia e investigación que contribuyan a la transformación de las relaciones entre la sociedad y el medio, y fomenten la prevención y resolución de problemas causantes de la insostenibilidad.

- Mejorar la interacción entre la demanda social de expertos/as en sostenibilidad en cualquier ámbito y la formación de licenciados/as y diplomados/das de la universidad

- Desarrollar modelos curriculares flexibles de enseñanza que faciliten la perspectiva holística del desarrollo humano ambiental y socialmente sostenible

- Reforzar el rol de las universidades y demostrar su capacidad de educar para conseguir un presente y futuro sostenibles.

- Pero, estos objetivos precisan de unos requisitos que podemos resumir en:

- No es posible conseguir los objetivos para la sostenibilidad en un espacio cerrado e impermeable.

- Es necesario trabajar en equipos y en red para favorecer una mayor diversidad de contextos de investigación, acción e interacción.

- Es importante conseguir progresos reales en los contenidos de la formación para la sostenibilidad, para crear y desarrollar respuestas y estrategias con un alto nivel de posibilidad de transferencia y validez en las universidades 0 instituciones de enseñanza superior. 
- Los procesos de gestión y de formación han de basarse en la investigaciónacción participativa, entendida como un proceso colaborativo en el que los gestores e investigadores puedan combinar investigación, aprendizaje, reflexión y acción.

Diversos autores coinciden en estos objetivos y requisitos, aportando sus experiencias desde diversas partes del mundo, por ejemplo, en Australia Sherren (2006) y Sibbel (2008); en los países nórdicos, Axelsson et al. (2008); en Estados Unidos, Davies et al. (2003); y Lozano (2006) en el Reino Unido.

Nuestro equipo de investigación persigue la introducción de la sostenibilidad en los estudios universitarios, lo que se ha denominado la Ambientalización Curricular, que, según la definición de la red ACES (Geli, A.M. 2004), es: "es un proceso continuo de producción cultural dirigido a la formación de profesionales comprometidos con la búsqueda permanente de las mejores relaciones posibles entre la sociedad y el medio ambiente, teniendo en cuenta los valores relacionados con la sostenibilidad: justicia, solidaridad, equidad, y el respeto a las diversidades tanto biológicas como culturales" 3 .

En el ámbito universitario este proceso incluye decisiones políticas de la institución que permitan generar los espacios necesarios para la participación democrática de los diversos estamentos internos en la definición de las estrategias institucionales y en el fomento de normas de convivencia que respondan a los objetivos y valores mencionados.

Los diseños curriculares han de incluir contenidos, metodologías, y prácticas sociales que preparen explícitamente en las competencias sostenibilizadoras que se especifiquen en el perfil de los títulos de las diferentes carreras.

Ello implica la formación de profesionales que puedan vivenciar situaciones reales que propicien la reflexión sobre las dimensiones afectivas, estéticas y éticas de las relaciones interpersonales y con el medio.

Requiere la realización de trabajos que faciliten el contacto con los problemas socioambientales en el propio escenario en el que ocurren; estas vivencias han de contemplar el análisis y la reflexión crítica sobre las alternativas de intervención en las relaciones entre la sociedad y el medio, orientando, propiciando y defendiendo el desarrollo humano ambiental y socialmente sostenible (Aznar, 2006; Esteban Chapapria, 2004)

Una forma complementaria de conseguir estos objetivos es la introducción en los planes de estudio de una asignatura cuyo contenido sea Educación para la Sostenibilidad o (Educación para el Desarrollo Sostenible), tendencia que se está extendiendo en las universidades europeas, sobretodo en las escuelas de Magisterio (Corney y Reid, 2007; Firth y Winter, 2007). En este caso hay que tener un especial cuidado en que el resto de asignaturas no dejen de contemplar las competencias para la sostenibilidad, ya que, tal y como indica Holmberg y Samuelsson (2006), ambas cosas son necesarias.

${ }^{3}$ Para más información sobre la red ACES, ver Junyent, M. y de Ciurana, A. M. Geli, (2008). Education for sustainability in university studies: a model for reorienting the curriculum. British Educational Research Journal 34 (6),763-782. 
Teniendo en cuenta que las universidades europeas están aplicando en sus reformas de los planes de estudios, dentro del marco del Espacio Europeo de la Educación Superior, modelos de formación por competencias, la metodología de trabajo considerada pertinente es la introducción de la sostenibilidad basada en la formación orientada al aprendizaje por competencias para la sostenibilidad; entendidas éstas como el conjunto complejo e integrado de conocimientos, procedimientos, actitudes y valores que los sujetos ponen en juego en los diferentes contextos en los que interactúan para resolver situaciones relacionadas con la problemática ambiental desde criterios de sostenibilidad. En este sentido una educación para la sostenibilidad debería contemplar la formación de competencias en estos tres ámbitos:

- Cognitivo: Competencias cognitivas relacionadas con el saber y vinculadas con la comprensión crítica de la problemática ambiental global y local;

- Metodológico: Competencias metodológicas, relacionadas con el saber hacer, la adquisición de habilidades, estrategias, técnicas y procedimientos para la toma de decisiones y la realización de acciones relacionadas con el medio ambiente y el desarrollo sostenible;

- Actitudinal: Competencias actitudinales, relacionadas con el saber ser y valorar, donde el desarrollo de actitudes y valores de sostenibilidad resulta imprescindible.

Desde la óptica de una actuación integral, la sostenibilización institucional tendría como finalidad la conformación de una Agenda 21 para la Universidad, en correspondencia con el desarrollo de las Agendas 21 locales. Coherentemente con ello, tendría que atender acciones a diferentes niveles: Gestión, Formación, Investigación y Participación, tal y como lo entienden autores como Capdevila (1998), Sterling (2005) o UII (2008). La integración efectiva de estos diferentes niveles de actuación en las instituciones de educación superior depende de una amplia gama de factores ubicados en diferentes ámbitos de concreción: macroscópico, microscópico y estratégico (Aznar y UII, 2009).

\section{RESULTADOS}

En los análisis realizados en este trabajo nos hemos centrado en el que hemos denominado "Ámbito macroscópico" que hace referencia a iniciativas desarrolladas en el ámbito internacional, estatal, regional y local. Este ámbito involucra cuestiones político-administrativas en las que son factores fundamentales: el apoyo institucional y el desarrollo de políticas educativas orientadas a fomentar la educación para el desarrollo sostenible (EDS).

Estos factores se deberían dar a diferentes niveles; en este trabajo se ha analizado el nivel internacional, centrándonos en Europa y el nivel estatal, con especial mención a aquellos países europeos que más han avanzado en la EDS y en lo que está ocurriendo en España. 


\section{Nivel Internacional}

Los organismos internacionales se están implicando en el desarrollo de la formación superior para la sostenibilidad a través de directivas, iniciativas, planes y programas de apoyo. En este plano se sitúa:

A. La iniciativa de Naciones Unidas y la promoción a través de la UNESCO de la Declaración de la Década de la Educación para el Desarrollo Sostenible 2005-2015. Y, en Europa, a través del documento Bolonia Sostenible: "Recomendaciones para la introducción del Desarrollo sostenible en el área de la Educación Superior en Europa. Guía para las instituciones de educación superior en relación con el proceso de Bolonia"; documento aprobado en 2005 por la Red Copernicus que reúne a 328 universidades europeas, que contiene propuestas para avanzar en la introducción de la sostenibilidad hasta 2012.

B. Los Regional Centres of Expertise $(R C E)$, creados para la implementación de la Educación para el Desarrollo Sostenible bajo el amparo de la United Nations University (UNU). En total existen en la actualidad 62 RCE en todo el mundo, de los cuales 17 están en Europa, siendo algunos de ellos muy activos en su relación con las universidades del entorno, que muchas veces son el núcleo a partir del cual se han creado y lideran la introducción de la sostenibilidad en la educación superior. Por ejemplo, el RCE de Skane, (Suecia) en el que participan las universidades de Lund y Mälmo. En Suecia todas las universidades tienen asignada, desde el 1 de febrero 2006, una tarea adicional mediante la Enmienda al "Act on Higher Education (1992) que indica que las instituciones de educación superior deben promover el desarrollo sostenible en sus actividades lo que significa que la presente y futuras generaciones tengan asegurados un medio ambiente saludable y un bien estar económica y socialmente justo. Esto significa que la educación y la investigación en las universidades dirigida hacia la educación para el desarrollo sostenible, es uno de las campos prioritarios en la educación y la investigación de la sociedad sueca (Axelsson et al, 2008).

C. El Programa de Educación para el Desarrollo Sostenible (ESD Programme) de la Universidad de la Naciones Unidas (UNU) en el que está colaborando muy estrechamente la Ubuntu Alliance, que tiene una amplia lista de miembros entre los cuales debemos destacar The International Association of Universities (IAU) y la University Leaders for Sustainable Future (ULSF); ambas asociaciones junto con Copernicus Campus y la UNESCO forman la Global Alliance to promote Higher Education for Sustainable Development (GHESP), basada en la Declaración de Lüneburg (2001), y que han desarrollado un Plan de Acción (2005). Uno de los Objetivos de la GHESP es promover el mayor entendimiento y un desarrollo más efectivo de las estrategias para la incorporación del desarrollo sostenible en las universidades y otras instituciones de educación superior. El énfasis debe ponerse en la necesidad de una aproximación interdisciplinaria tanto en la docencia como en la investigación.

D. El Programa Erasmus de la Unión Europea: se ha publicado un informe sobre "Education for Sustainable Development In the European Union -Education Programmes COMENIUS; ERASMUS; LEONARDO DA VINCI \& GRUNDTVIG" (Anne 
Busch, 2007) del Institute for Environmental \& Sustainability Communication de la Universidad de Lüneburg. El informe hace un Inventario del coste invertido en Educación para el Desarrollo sostenible en los programas de educación de la Unión Europea así como una recopilación de Proyectos de Buenas Prácticas en educación para el desarrollo sostenible (EDS) en los Programas de Educación subvencionados por la Unión Europea. El primer problema al abordar estas cuestiones, tal y como indica Busch, son los importantes déficits en los Programas de Desarrollo de la UE en documentación de proyectos; por una parte, no existe una base de datos en la que se pueda realizar una búsqueda temática por temas específicos y de referencia. A causa de esta estructura, no es posible una búsqueda temática en las bases de datos, en la que se pueda comentar un proyecto, un tema no cubierto o hacer un balance definitivo viendo los valores incluidos en la EDS en el marco de referencia de los programas de Educación de la UE. Por otra parte, es especialmente difícil, en los Programas de Educación, separar la EDS, de la integración social, cooperación internacional y políticas de innovación. Por ello, la autora del informe ha considerado sólo los proyectos en los que se menciona Educación para el Desarrollo Sostenible/Agenda 21 explícitamente en el título.

Estos mismos problemas nos hemos encontrado nosotros en este trabajo, pero centrándonos en la introducción de la sostenibilidad en la Educación Superior; la inmensa cantidad de información diseminada en la bibliografía y en la red hace muy difícil una visión de conjunto del estado de la cuestión.

Tal como indica Busch en su informe, en lo que respecta al Programa ERASMUS (Educación Superior), éste es, de lejos, el programa de becas más amplio a nivel mundial que promueve el aprendizaje y la enseñanza en el sector de la educación superior, a través de la movilidad cruzada, con la participación de más de 2000 universidades de 31 países de la Unión Europea, que suponen unos 1.5 millones de estudiantes y más de 100000 profesores.

El análisis de la Educación para el Desarrollo Sostenible en el marco del programa ERASMUS ha aportado los siguientes datos:

En la Acción 1 del Programa: Colaboración de universidades europeas, tenemos por una parte los Programas intensivos, y por otra, los Proyectos de desarrollo del currículum.

\section{Programas intensivos}

a) En 2003 unos 12 de los 175 programas se centraban en la sostenibilidad, entre ellos se incluyen programas de: Agricultura Biológica, Moving School 21-Seminario interdisciplinar en Orientación Nutricional, y planeamiento de desarrollo urbano pensado para niños y jóvenes en concordancia con la Agenda 21, ECOPOLIS: Planeamiento Sostenible y principios de diseño y Planeamiento Urbano y regional para entornos sostenible

b) En 2005, 15 de los 175 programas mencionaban, tanto implícita como explícitamente, "sostenibilidad" en su título, y muchos de los programas estaban relacionados con integración, exclusión social, y uso de recursos, tales como: SINEXInclusión y Exclusión Social en Educación, Resiliencia, Vulnerabilidad y sostenibilidad 
en entornos rurales europeos, NESSIE: Valorización de agro-recursos y Desarrollo Sostenible Parte 1: Gestión de la Calidad del Agua y Medio Ambiente.

\section{Proyectos de Desarrollo del Currículum}

Existe un potencial especial en el marco de la UE para desarrollar proyectos sobre enseñanza y aprendizaje sostenible especialmente a través de la unión de universidades en el desarrollo del currículum. De los 33 proyectos desarrollados en 2004 siete daban especial relevancia a la sostenibilidad ${ }^{4}$

En la Acción 2 se contemplan las ayudas a la movilidad de profesores y estudiantes.

En la Acción 3 del Programa Erasmus, referida a Redes Temáticas, existen en la Agencia Ejecutiva de la UE un total de nueve redes temáticas para discusiones, las cuáles integran aspectos de sostenibilidad en mayor o menor grado. A la vez, estas redes muestran una voluntad de permanencia y planes para tener un papel en el futuro. Estos programas incluyen:

- HUMANITARIANNET Thematic Network on Humanitarian Development Studies (2001- 2009)

- LE NOTRE: Landscape Education-New Opportunities in Teaching and Research in Europe (2004-2009)

- The Consumer Citizenship Network (2004-2009)

- ISEKI Food: Integrating Safety and Environment Knowledge in Food Towards European Sustainable Development (2002-2008)

E. Otras redes: Hemos encontrado muchas referencias a redes temáticas de universidades europeas conjuntamente con universidades latinoamericanas y de otras partes del mundo, traemos como ejemplo la ACES network ("Reorienting the curricula toward sustainability plan of higher studies offered in each Institutions of the ACES net), que integra 10 universidades $y$, que como su nombre indica se han centrado en la introducción de la sostenibilidad en los currícula. ${ }^{5}$

\section{Nivel Nacional}

Únicamente nos referiremos a algunos países europeos, en los que más ha avanzado la introducción de la EDS en las universidades, a título de ejemplo

Suecia:

Sin ninguna duda Suecia es el país más avanzado en la implementación de la EDS, ya hemos citado la enmienda que desde 2006 obliga a todas las universidades a promover el desarrollo sostenible en sus actividades.

\footnotetext{
${ }^{4}$ Consultar Busch (2007) para más información sobre estas redes

${ }^{5}$ Los componentes de la Red ACES son: Cataluña (España): Universitat de Girona y Universitat Autònoma de Barcelona; Alemania: Technische Universität Hamburg-Harburg Technologie GmbH (TuTech); Italia: Universita' degli Studi del Sannio: Portugal: Universitye de Aveiro; Argentina: University de San Luis and University Nacional de Cuyo; Brasil: Universidade Estadual de Campinas, Universidade Estadual Paulista - Río Claro and Universidade Federal de Sao Carlos
} 
Hemos citado anteriormente la Lünd University, una de las pioneras en estos procesos y no podemos dejar de mencionar la Chalmers University of Thecnology ${ }^{6}$ y su Center for Environment and Sustainability, GMV, compartido con la University of Gothenburg. Ambas han editado The Gothenburg Recommendations on Education for Sustainable Development (nov. 2008), donde se defiende que la Educación para el Desarrollo Sostenible debe impregnar los currícula, en los documentos y en los materiales pedagógicos; lo cual incluye una necesaria revisión del currículum y el desarrollo de nuevos currícula. La reorientación en la educación requiere de enfoques multi-, inter- y transdisciplinarios en el desarrollo del currículum, para extender los enfoques que cada disciplina aporta al conocimiento. Estos enfoques pueden propiciar otras formas de conocimiento de las que existen en el currículum formal.

\section{Holanda:}

La Red Nacional Holandesa para la introducción del desarrollo sostenible en los planes de estudio de educación superior (DHO), fue creada en 1998 y es una fundación independiente financiada por el gobierno. La mayoría de los grupos de la red se coordinan desde el Centro de Especialización para el Desarrollo Sostenible (ECDO) de la Universidad de Amsterdam. La DHO pretende crear oportunidades, entornos y metodologías de aprendizaje innovadores en la educación superior, que permitan a los individuos desarrollar sus competencias en el campo del desarrollo sostenible (DS). Esto significa que los estudiantes pueden adquirir:

1. Una nueva percepción del concepto de DS

2. Una nueva percepción de aquello en lo que su disciplina puede contribuir al DS

3. Las competencias para colaborar con otros estudiantes de otras disciplinas

4. Las competencias para desarrollar estrategias de trabajo en torno al DS en sus futuras carreras profesionales.

No podemos dejar de mencionar entre las universidades holandesas la Delft University of Technology ${ }^{7}$, otra de las pioneras en Europa en atender la educación para la sostenibilidad.

Reino Unido:

Trabajando conjuntamente con otras instituciones, la Higher Education Funding Council for England (HEFCE) promueve y subvenciona la alta calidad y la buena relación costes-efectividad, tanto en la docencia como en la investigación, atendiendo las diversas necesidades de los estudiantes, la economía y la sociedad. HEFCE está formada por 130 instituciones de educación superior y 124 colleges

\footnotetext{
${ }^{6}$ Citamos de su web "Chalmers' ambition to contribute to sustainable development is based on the work carried out in departments in the form of research and teaching. Many of the great questions of the future to which Chalmers wishes to contribute by developing knowledge and technology in the form of teaching and research deal with both environmental questions and the broader perspective of sustainable development. Here we are to a great extent dependent on an interplay between many different disciplines both within and outside Chalmers. To help us we have several different centres and networks."

http://www.chalmers.se/en/sections/about_chalmers/environmental-science1402

${ }^{7}$ http://home.tudelft.nl/en/
} 
En julio de 2005 HEFCE publicó 'Sustainable development in higher education' (HEFCE $2005 / 28)^{8}$ donde se resume el acercamiento a la promoción de una agenda para el desarrollo sostenible. Contiene un planteamiento estratégico hasta 2015 y un plan de acción.

En 2005, HEFCE dio una visión de como los centros de educación superior pueden contribuir al desarrollo sostenible. Se plantea como cada una de las universidades y colleges pueden contribuir tanto como centros de enseñanza y de investigación como desde la gestión de los campus, así como desde el papel que cumplen sus trabajadores como protagonistas principales en la comunidad local. Puede consultarse el Plan HEFCE "Sustainable development in higher education Consultation 2008 update to strategic statement and action plan" en la red, donde se constata que existen 74 Centers for Excellence in Teaching and Learning (CETLs) ${ }^{9}$ en los que se pueden encontrar prácticas de excelencia en la docencia. Además existen el Centre for Sustainable Futures ${ }^{10}$ y el Centre for Sustainable Communities, que a través del Integrated Professional Education (C-SCAIPE) ${ }^{11}$ centran su visión en la educación para el desarrollo sostenible. El primero ha desarrollado acercamientos a la sostenibilidad para cientos de estudiantes de la Universidad de Plymouth, desarrollando una amplia política de sostenibilidad, introduciendo cursos y masters y dando apoyo a los cambios en los currícula de las escuelas locales. El segundo, C-SCAIPE en la Universidad de Kingston aspira a formar graduados con una formación holística en comunidades sostenibles, que sean capaces de trabajar en la creación de las mismas.

La Comisión Nacional del Reino Unido para la UNESCO está siguiendo la implementación de la Década en el Reino Unido y la HEFCE es miembro de su grupo de trabajo en Educación para el Desarrollo Sostenible. La región de East Midlands fue la primera en el Reino Unido en ser designada por las Naciones Unidas como un Regional Centre of Expertise (RCE) en EDS ${ }^{12}$. Las universidades son el centro de estas iniciativas y existen otros dos RCEs en Reino Unido: uno en el Noreste, liderado por la Universidad de Newcastle, y otro en West Midlands, liderado por la Universidad de Gloucestershire.

\section{Alemania:}

En el año de 2004 el Prof. Dr. Gerhard de Haan fue elegido Presidente del Comité Nacional Alemán de la Década de las Naciones Unidas para la "Educación para un Desarrollo Sostenible". Hasta el año de 2014, el catedrático berlinés coordina algunos centenares de instituciones y expertos pertenecientes a la "Alianza para un aprendizaje sostenible" y recoge sus propuestas de iniciativas educativas ${ }^{13}$.

En el marco de la Década, se ha aprobado la "Recommendation of the Standing Conference of the Ministers of Education and Cultural Affairs of the Länder of the Federal Republic of Germany (KMK) y la Comisión alemana de la UNESCO (DUK) de la

\footnotetext{
8 www.hefce.ac.uk under Publications.

${ }^{9}$ Para más información ver: www.hefce.ac.uk under Learning \& teaching/Teaching initiatives.

${ }^{10}$ Para más información sobre el Centre for Sustainable Futures, ver www.csf.plymouth.ac.uk

${ }^{11}$ Para más información sobre C-SCAIPE, ver www.kingston.ac.uk/surveying

${ }^{12}$ For more information see www2.le.ac.uk under Projects/RCEEM.

${ }^{13}$ http://www.goethe.de/ges/umw/dos/nac/ler/es3113206.htm
} 
Educación para el Desarrollo Sostenible (EDS) 2006" que proporciona a las escuelas recomendaciones prácticas y un marco útil para la implementación de la EDS.

También las universidades alemanas se han implicado en la EDS, a partir de la Conferencia de Berlín de 2004, la Conferencia alemana de Rectores (HRK) creó, con financiación del Ministerio de Educación y Ciencia, el Centro de Servicios y el Centro de Excelencia para Bolonia, para dar soporte y asistencia a las instituciones de enseñanza superior alemanas en el proceso de reforma; a la convocatoria pública respondieron 127 universidades y la Conferencia de Rectores (HRK) seleccionó las 22 universidades que presentaron las mejores propuestas (Zervakis y Wahlers, 2007).

La Universidad de Lüneburg es una de las pioneras en EDS, de hecho allí se firmó en 1997 la declaración que lleva su nombre sobre la promoción de la Agenda 21 para las universidades y ha sido una de las pioneras en implantar energía solar, hacer público su balance de $\mathrm{CO}_{2}$ y en investigar e impartir masters sobre sostenibilidad.

En Hamburgo encontramos otro de los Regional Centre of Expertise en EDS, liderado por la Universidad de Ciencias Aplicadas de Hamburgo y con una amplia implantación social en la región de Hamburgo (Filho y Schwarz, 2008)

España:

En septiembre de 2002 la Asamblea de la Conferencia de Rectores de las Universidades Españolas (CRUE) aprobó por unanimidad la creación del "Grupo de Trabajo de la CRUE sobre la Calidad Ambiental y el Desarrollo Sostenible en las universidades españolas". A este grupo de trabajo se han adscrito 24 universidades y fue auspiciado por algunas de las universidades que más tiempo y esfuerzo han dedicado a estos temas (Univ. Politécnica de Cataluña, Univ. Autónoma de Madrid, Univ. Autónoma de Barcelona, Universidad de Girona...).

La CRUE en sus Directrices para la Sostenibilización Curricular ${ }^{14}$ recomienda algunas actuaciones específicas a promover por parte de las autoridades competentes en materia de regulación de las titulaciones oficiales; se trata de actuaciones que garanticen:

- La revisión integral de la currícula desde la perspectiva del Desarrollo Sostenible que asegure la inclusión de los contenidos transversales básicos en sostenibilidad en todas las titulaciones, con el fin de adquirir las competencias profesionales, académicas y disciplinares necesarias. Lo anterior debe lograrse mediante el reconocimiento académico cuantificable de contenidos generales de sostenibilidad para todas las titulaciones y de contenidos específicos adaptados al contexto de cada titulación.

- La inclusión de criterios de sostenibilidad en los sistemas de evaluación de la calidad universitaria.

14 Documento aprobado por el Comité Ejecutivo del Grupo de Trabajo de Calidad Ambiental y Desarrollo Sostenible de la CRUE, celebrado en Valladolid el 18 de abril de 2005.y revisado y reafirmado en la reunión de Granada en marzo de 2009 
- La inclusión de criterios de sostenibilidad en el proceso de evaluación del profesorado, con el fin de asegurar una docencia coherente con los principios de Desarrollo Sostenible".

Con respecto al primer punto, se ha avanzado en este tema en estos últimos cuatro años, y muestra de ello es que se han incluido en la legislación española cuestiones importantes:

a) La Ley Orgánica 4/2007 de Universidades de 12 de abril, por la que se modifica la Ley Orgánica 6/2001, sienta las bases para realizar una modernización de la Universidad española (Título VI), y se desarrolla con el Real Decreto 1393/2007, de 29 de octubre, por el que se establece la ordenación de las enseñanzas universitarias oficiales.

b) En el RD 1393/2007 se especifica en el preámbulo que "se debe tener en cuenta que la formación en cualquier actividad profesional debe contribuir al conocimiento y desarrollo de los Derechos Humanos, los principios democráticos, los principios de igualdad entre mujeres y hombres, de solidaridad, de protección medioambiental, de accesibilidad universal y diseño para todos, y de fomento de la cultura de la paz"

c) En las Resoluciones de cada Titulación aparecen las Condiciones a las que deberán adecuarse los planes de estudios conducentes a la obtención de títulos que habiliten para el ejercicio de la profesión regulada correspondiente y también se han publicado las Órdenes correspondientes para la Verificación de los títulos oficiales que habiliten para el ejercicio de la profesión regulada.

Nuestro grupo de investigación ha realizado el seguimiento de todas estas Resoluciones y Órdenes en cuanto a la especificación de competencias para la sostenibilidad (Martínez-Agut et al, 2009).

La revisión se ha realizado sobre las Órdenes de Verificación de los títulos, veamos como ejemplo el título de Ingeniero de Caminos, Canales y Puertos

Entre los Objetivos de este título se encuentra.

$\checkmark$ Comprensión de los múltiples condicionamientos de carácter técnico, legal y de la propiedad que se plantean en el proyecto de una obra pública, y capacidad para establecer diferentes alternativas válidas, elegir la óptima y plasmarla adecuadamente, previendo los problemas de su construcción, y empleando los métodos y tecnologías más adecuadas, tanto tradicionales como innovadores, con la finalidad de conseguir la mayor eficacia $y$ favorecer el progreso y un desarrollo de la sociedad sostenible y respetuoso con el medio ambiente.

$\checkmark$ Capacidad para planificar y gestionar recursos hidráulicos y energéticos, incluyendo la gestión integral del ciclo del agua.

$\checkmark$ Capacidad para la realización de estudios de planificación territorial, del medio litoral, de la ordenación y defensa de costas y de los aspectos medioambientales relacionados con las infraestructuras 
$\checkmark$ Capacidad de realización de estudios, planes de ordenación territorial y urbanismo y proyectos de urbanización.

$\checkmark$ Capacidad para evaluar y acondicionar medioambientalmente las obras de infraestructuras en proyectos, construcción, rehabilitación y conservación.

$\checkmark$ Capacidad para proyectar y ejecutar tratamientos de potabilización de aguas, incluso desalación, y depuración de éstas. Recogida y tratamiento de residuos (urbanos, industriales o incluso peligrosos).

$\mathrm{Y}$ entre las Competencias que deben adquirir los futuros ingenieros:

- Capacidad para analizar y diagnosticar los condicionantes sociales, culturales, ambientales y económicos de un territorio, así como para realizar proyectos de ordenación territorial y planeamiento urbanístico desde la perspectiva de un desarrollo sostenible

Como puede verse en esta titulación está muy presente la perspectiva sostenibilista. No es así en todas las que hemos revisado, pero si se constata un avance en la inclusión de objetivos y competencias para la sostenibilidad. Ahora las universidades españolas están introduciendo estos requerimientos en sus nuevos planes de estudio.

Entre las universidades españolas, hay que hacer una mención especial de la Universidad Politécnica de Cataluña (UPC), otra de las universidades pioneras en Europa en la introducción de la educación para la sostenibilidad

Y también es necesario mencionar la Red de investigación en Educación para la Sostenibilidad (Edusost) ${ }^{15}$. En 2003, y gracias al soporte del Departamento de Universidades, Investigación y Sociedad de la Información del Gobierno de Cataluña, se creó la Web de Ambientalización curricular de Educación Superior (ACEU), red que está integrada por grupos de investigación en educación para la sostenibilidad, a la que pertenecen las universidades de la red pública catalana. En particular, Universitat de Girona, Universitat Politècnica de Catalunya y Universitat Autònoma de Barcelona Los grupos que integran la Red vieron la necesidad de darle continuidad en una esfera más amplios de aplicación del conocimiento, esto implicó la creación de una nueva red, más participativa y capaz de acoger otras instituciones educativas, empresas y administraciones, que se denomina Edusost.

\section{Las Competencias Sostenibilizadoras en los nuevos planes de estudio españoles}

A la luz de esta legislación hemos revisado los planes de estudio que se han sometido a información pública en nuestra universidad, hasta el momento 41 planes de grado. Nuestro grupo ha presentado informe y/o alegaciones a todos ellos, atendiendo sobretodo a las Competencias Generales de los diferentes títulos de Grado. Ya que, entendemos que deben recoger lo especificado en el RD antes citado y a la concreción de esas Competencias Generales en las diferentes materias y asignaturas y en sus competencias específicas. (Ull et al, 2009)

\footnotetext{
${ }^{15}$ http://edusost.cat/content/view/12/27/lang,en/
} 
Si los nuevos planes no las recogen se han presentado propuestas para que se incluyan de manera que en todas las titulaciones aparezcan las competencias para la sostenibilidad.

Citaremos como ejemplo dos títulos que si las recogían: el Título de Graduado/a en Biología por la Universitat de Valencia, en el que se recogen como Competencias Generales Transversales:

$\checkmark$ Comprensión de la naturaleza biológica del ser humano y de las implicaciones de ésta en la igualdad de derechos entre géneros y grupos culturales

$\sqrt{ }$ Desarrollo de actitudes y valores de sostenibilidad

Y como Competencias Específicas:

(1) Conocer la diversidad de los seres vivos

(2) Conocer la diversidad de los ecosistemas

(3) Comprender la problemática ambiental global, regional y local.

En el Título de Graduado/a en Biotecnología por la Universitat de Valencia se recogen, entre otras, las siguientes Competencias Generales:

- Comprensión del mundo natural como producto de la evolución y de su vulnerabilidad frente a la influencia humana

- Reconocimiento, respeto y promoción de los derechos humanos fundamentales, especialmente los de igualdad, de los valores democráticos, de los valores de sostenibilidad y de los valores propios de una cultura de paz

Y como Competencia específica:

- Comprender el papel del profesional en Biotecnología en el contexto científico y social.

Ahora estas competencias deben de ser desarrolladas en las diferentes materias que componen la titulación correspondiente y que son susceptibles de contemplar estas competencias.

En otras universidades españolas se está discutiendo la aprobación de un corto catálogo de asignaturas transversales, comunes a todas las titulaciones, entre las que se encuentra la EDS.

De una manera u otra, como decíamos al principio lo importante es que los estudiantes hayan recibido formación en EDS para que cuando estén ejerciendo sus profesiones sepan hacerlo de una manera sostenible.

\section{CONCLUSIONES}

En la Declaración de Bonn realizada por los participantes en la Conferencia Mundial de la UNESCO sobre la educación para el desarrollo sostenible, celebrada en Bonn (Alemania, abril 2009), se hace una valoración de la evolución de los primeros cinco años de la Década en la que se subraya que la EDS: 
- está imprimiendo una nueva dirección a la enseñanza y el aprendizaje para todos. La EDS promueve la educación de calidad e integra a todos sin excepción. Se basa en valores, principios y prácticas necesarios para responder eficazmente a los retos actuales y futuros.

- ayuda a las sociedades a hacer frente a las diferentes prioridades y problemas entre otros, los relativos al agua, la energía, el cambio climático, la atenuación del riesgo y los desastres, la pérdida de la biodiversidad, la crisis alimentaria, las amenazas contra la salud, la vulnerabilidad social y la inseguridad. La EDS es esencial para el surgimiento de nuevas ideas sobre la economía y contribuye a crear sociedades resistentes, saludables y sostenibles, mediante un enfoque sistémico e integrado. Además, confiere nueva pertinencia, calidad, significado y finalidad a los sistemas de enseñanza y formación, y propicia la intervención de los medios educativos formal, no formal e informal y de todos los sectores sociales en un proceso de aprendizaje a lo largo de la vida.

- se basa en valores de justicia, equidad, tolerancia, suficiencia y responsabilidad. Promueve la igualdad entre hombres y mujeres, la cohesión social y la reducción de la pobreza, y asigna un lugar prioritario al cuidado, la integridad y la honradez, como se enuncia en la Carta de la Tierra. La EDS se sustenta en principios propicios a modos de vida sostenibles, la democracia y el bienestar de los seres humanos. Proteger y restaurar el medio ambiente, conservar los recursos naturales y utilizarlos de manera sostenible, actuar ante las pautas de consumo y de producción no sostenibles y crear sociedades justas y pacíficas son también principios importantes en los que se funda la EDS.

- Y hace hincapié en los enfoques creativos y críticos, la reflexión a largo plazo, la innovación y la autonomía para afrontar la incertidumbre y solucionar problemas complejos. La EDS pone de relieve la interdependencia entre el medio ambiente, la economía, la sociedad y la diversidad cultural, desde el ámbito local hasta el mundial, y tiene en cuenta el pasado, el presente y el futuro. La EDS, vinculada a las necesidades y condiciones específicas de vida de la población, ofrece las competencias para hallar soluciones y aprovecha las prácticas y los conocimientos existentes en las culturas locales, así como en las nuevas ideas y tecnologías.

En relación a los logros conseguidos, numerosos países han realizado progresos en la implementación de la EDS y han elaborado marcos de políticas innovadoras. Diversos organismos de las Naciones Unidas, organizaciones no gubernamentales (ONG), órganos regionales y redes de colaboración participan en actividades concretas en apoyo de ámbitos específicos de la EDS. Están en curso los esfuerzos para mejorar la comprensión, promoción, aplicación y evaluación de la calidad de la EDS. Se ha elaborado un marco de seguimiento y evaluación a escala mundial.

Y en relación a la formulación de políticas en los Estados Miembros, en el terreno práctico se propone: 
- apoyar la incorporación de temas del desarrollo sostenible, mediante una estrategia integrada y sistémica, en todos los niveles de la educación formal, no formal e informal, en particular mediante la elaboración de métodos pedagógicos eficaces, la formación de docentes, las prácticas de enseñanza, los planes y programas de estudio, los materiales didácticos y el fomento del liderazgo en el sector educativo, y también mediante el reconocimiento de la significativa contribución de la educación no formal y el aprendizaje informal, la formación profesional y el aprendizaje en el lugar de trabajo. El desarrollo sostenible es un tema transversal importante para todas las disciplinas y todos los sectores.

- Reorientar los programas de elaboración de planes de estudio y formación de docentes, con miras a integrar la EDS en los programas de formación inicial y de perfeccionamiento para docentes en funciones. Alentar a las instituciones de formación de docentes, los maestros y los profesores a crear redes y a elaborar e investigar las prácticas pedagógicas idóneas. En particular, ayudar a los docentes a formular estrategias de EDS aplicables a clases numerosas y a evaluar los procesos de aprendizaje de la EDS.

- Promover el diálogo sobre políticas de base empírica acerca de la EDS, aprovechando las estrategias pertinentes de investigación, seguimiento y evaluación y el intercambio y reconocimiento de las prácticas ejemplares. Crear indicadores nacionales de EDS que contribuyan a la aplicación eficaz y la evaluación de los resultados y procesos en la materia.

- Establecer y ampliar iniciativas de colaboración sobre la EDS, para integrarla en la capacitación, la formación profesional y el aprendizaje en el lugar de trabajo, mediante la participación de la sociedad civil, los sectores público y privado, las ONG y los asociados en el desarrollo. La EDS debería ser parte integrante de la formación de los dirigentes de empresas, industrias, sindicatos, organizaciones voluntarias y sin fines de lucro, y de los servicios públicos. Reorientar los programas de enseñanza y formación técnica y profesional a fin de que incorporen a la EDS.

- Producir conocimientos mediante la creación de redes de EDS. Individualizar y apoyar a las escuelas, universidades y otras instituciones de educación superior e investigación, centros de enseñanza y redes de educación que puedan servir como centros de competencias e innovación que generen y compartan conocimientos, y creen recursos para la EDS. Examinar el potencial de sitios geográficos y biorregionales específicos que podrían constituir "laboratorios" delimitados territorialmente para la EDS.

- Alentar y fortalecer la excelencia científica, la investigación y la creación de nuevos conocimientos para la EDS mediante la participación en ésta de las redes de establecimientos de educación superior e investigación. Movilizar las funciones esenciales de las universidades: docencia, investigación y participación comunitaria, con el fin de fortalecer el conocimiento mundial y local de la EDS y utilizar en este proceso las Cátedras UNESCO sobre EDS y las redes del programa de la UNESCO. Crear estructuras institucionales y 
organizativas que faciliten la flexibilidad, la participación estudiantil y los programas pluridisciplinarios, y generar proyectos piloto capaces de responder a la complejidad y la urgencia de la EDS. También deberían crearse y aplicarse dispositivos para recompensar las iniciativas y la investigación en materia de EDS en la enseñanza superior.

\section{REFERENCIAS BIBLIOGRÁFICAS}

Aznar Minguet, P. (2006). El reto educativo de la sostenibilidad en el marco europeo de la educación superior. En A. Escolano Benito, Cambio educativo y cultura de la sostenibilidad. (Madrid: Biblioteca Nueva).

Aznar Minguet, P y UII, M.A. (2009). La formación de competencias básicas para el desarrollo sostenible: el papel de la Universidad. Revista de Educación no extraordinario 2009, 219-237. Ministerio de Educación. http://www.revistaeducacion.mec.es/inicio.html

Axelsson, H., Sonesson, K y Wickenberg, P. (2008). Why and how do universities work for sustainability in higher education (HE)?. International Journal of Sustainability in Higher Education,9 (4),469-478

Busch, A. (2007). Education for Sustainable Development In the EU -Education Programmes Comenius; Erasmus; Leonardo da Vinci \& Grundtvig. Institute for Environmental \& Sustainability Communication de la Universidad de Lüneburg. Alemania

Capdevila i Peña, I. (1999). L'ambientalització de la universitat. Monografies d 'educació ambiental. No 6. Palma de Mallorca: Di7 Edició

Corney, G. y Reid, A. (2007). Student teachers' learning about subject matter and pedagogy in education for sustainable development. Environmental Education Research, 13(1), 33-54

Clugston, R. (2002). "Introduction", in Filho, W. (Ed.), Teaching Sustainability at Universities, Peter Lang, Frankfurt.

Davies, S, Edmister, J., Sullivan, K \& West, C.(2003). Educating sustainable societies for the twenty-first century. International Journal of Sustainability in Higher Education, 4 (2),169-179

Filho, W.L. y Schwarz, J. (2008). Engaging stakeholders in a Sustainability context. The regional Centre of Expertise on Education for Sustainable Development in Hamburg and región. International Journal of Sustainability in Higher Education 9 (4). 498-508.

Firth, R. y Winter, C. (2007). Constructing education for sustainable development. The secondary school geography curriculum and initial teacher training. Environmental Education Research, 13(5), 599-619.

Global University Network for Innovation (GUNI) (2008). La Educación Superior en el mundo 3. Educación Superior: Nuevos Retos y Roles Emergentes para el Desarrollo Humano y Social. Madrid: Ed.Mundi-prensa. 
Junyent, M. y de Ciurana, A. M. G. (2008). Education for sustainability in university studies: a model for reorienting the curriculum. British Educational Research Journal, 34 (6), 762-783.

Lozano García, F. J., Gándara, G., Perrni,O., Manzano,M y Hernandez, D. E. (2008). Capacity Building: a course on sustainable development to educate the educators". International Journal in Higher Education, 9 (3), 257-281.

Lozano, R. (2006).Incorporation and institutionalization of sustainable development into universities: breaking through barriers to change. Journal of Cleaner Production, 14 (9 y 11), 787-796.

Martinez Agut, M. P., Aznar, P., Ull, A. y Piñero, A. (2007). Promoción de la sostenibilidad en los currícula de la enseñanza superior desde el punto de vista del profesorado: un modelo de formación por competencias. Educatio Siglo XXI, 25, 187-208

Martínez Agut, M. P., Ull, M. A., Piñero, A., Palacios, B. y Aznar Minguet, P. (2008). Análisis de las referencias a la sostenibilidad en los nuevos títulos universitarios. VI Jornadas de investigación en docencia universitaria. Universidad de Alicante.

Martínez Agut, M. P., UII, M. A. y Aznar Minguet, P. (2009). Títulos que habilitan para el ejercicio de profesiones reguladas: análisis de las referencias a la sostenibilidad. VII Jornadas de investigación en docencia universitaria. Universidad de Alicante.

Sherren, K. (2006). Core issues: reflections on sustainability in Australian University coursework programs, International Journal of Sustainability in Higher Education, 7(4), 400-41.

Sibbel, A. (2009). Pathways towards sustainability through higher education. International Journal of Sustainability in Higher Education,10 (1), 68-82.

Sterling, S. (2005). Higher education, sustainability, and the role of systemic learning in Blaze Corcoran P.B. and Wals, A. E.J. (Eds) Higher Education and the Challenge of Sustainability: Problematics, Promise and Practice, Dordretch: Kluwer Academic Press

UII, M. A. (2008). El impacto de la actividad universitaria sobre el medio ambiente. Revista Eureka Enseñ. Divul. Cien., 5(3), 356.366. http://www.apaceureka.org/revista

UII, M. A., Martínez Agut, M. P. y Aznar Minguet, P. (2009). Competencias para la sostenibilidad en los planes de estudio de los nuevos títulos universitarios de grado. VII Jornadas de investigación en docencia universitaria. Universidad de Alicante.

UNESCO (1998). La educación superior y el desarrollo humano sostenible. La educación superior en el siglo XXI. Visión y acción. (Conferencia Mundial sobre la educación superior. París. ED 98/CONF 202/7.2).

UNESCO (2005). Decenio de las Naciones Unidas de la Educación para el Desarrollo 
Sostenible 2005-2014: Plan de aplicación internacional. Proyecto. (UNESCO.Paris).

Zervakis,P y Wahlers, M. (2007). Education for Sustainable Development and Bologna Proces-The implementation of the Bologna Process in Germany" BNEJournal.On line Magazine Education for Sustainable Development. http://bneportal.de/coremedia/generator/pm/en/Issue_001/01_Contributors/

\section{PÁGINAS WEB}

ACES Network:

http://insma.udg.es/ambientalitzacio/web alfastinas/angles/a index.htm

Action for a sustainable world UK: http://www.forumforthefuture.org/

Copérnicus Programme:

http://unece.org/env/esd/information/COPERNICUS\%20Guidelines.pdf

Federal Ministery for Education and Research (Germany):

http://www.fona.de/en/7284

UN Decade in Germany:

http://www.bnEportal.de/coremedia/generator/unesco/en/01 Home/English $\underline{20 H o m e p a g e . h t m l}$

GESHP: http://webapps01.un.org/dsd/partnerships/public/partnerships/71.html

Halifax Declaration: http://www.unesco.org/iau/sd/sd_declarations.html

Swansea Declaration: http://www.iisd.org/educate/declarat/swansea.html

Swedish National Agency for Higher Education:

http://www.hogskoleverket.se/2.539a949110f3d5914ec800056285.html

Talloires Declaration: http://www.unesco.org/iau/sd/sd_declarations.html

http://www.ulsf.org/about.html

Red de investigación en Educación para la Sostenibilidad (Edusost)

http://edusost.cat/content/view/12/27/lang,en/

UNU: http://www.ias.unu.edu/sub page.aspx? catID $=108 \& d d \mid I D=54$

UNU (Regional Centres of Expertise)

http://www.ias.unu.edu/sub_page.aspx?catID =108\&ddlID $=183$

World Conference 2009 Bonn

http://www.esd-world-conference-2009.org/en/whats-new/news-

detail/item/bonn-declaration-now-available-in-8-languages.htl 


\title{
ANALYSING OF INTRODUCTION OF SUSTAINABILITY INTO HIGHER EDUCATION IN EUROPE: INSTITUTIONAL ENGAGEMENTS AND CURRICULAR PROPOSALS
}

\begin{abstract}
SUMMARY
In the current context of the harmonization process of university curricula and the European Convergence Model for universities, the Bologna Declaration (the Sustainable Bologna Document) offers the idea of making higher education a sustainable area. From this viewpoint, this article, firstly analyses the legal regulations being established to verify whether the references to including sustainability criteria result in new curricula in European universities. Secondly, it analyses the activities of the existing collaboration networks among European universities (Copernicus, HBO, ULSF, ACES, etc.) which aim to introduce sustainability into higher education curricula. We believe that this analysis may contribute to an evaluation of the actions taken at the higher education level within the context of UNESCO's Decade of Education for Sustainable Development. The results obtained reveal that progress has been made in introducing sustainability criteria into educational laws and of the activities conducted by European universities, organized through powerful networks in recent years.
\end{abstract}

Key words: Sustainability; Higher Education; European level. 